\title{
IOT based Advance Traffic Management System with the Emergence of 5G: A Concise Review
}

\author{
Parv Kukreja \\ Student \\ Manav Rachna \\ International Institute of \\ Research and Studies \\ Faridabad, Harayana, \\ India
}

\author{
Aashutosh Vats \\ Student \\ Manav Rachna \\ International Institute of \\ Research and Studies \\ Faridabad, Harayana, \\ India
}

\author{
Anish Raj \\ Student \\ Manav Rachna \\ International Institute of \\ Research and Studies \\ Faridabad, Harayana, \\ India
}

\author{
Pratima Manhas, $\mathrm{PhD}$ \\ Associate Prof \\ Manav Rachna \\ International Institute of \\ Research and Studies \\ Faridabad, Harayana,
}

India

\begin{abstract}
This paper talks about what is $5 \mathrm{G}$, its technicalities, how it changes the networking in the near future and how $5 \mathrm{G}$ will bring a revolution in the world of Internet of Things. Then it focuses on the application of IoT in traffic management system. There are 3 basic steps to this process involving sensing of data, transfer of data through and on cloud and processing of the data. The camera capture an image sequence (video)which is sent to the cloud, information about the vehicles on the road is extracted from the data, this can be done through various methods, few of which are discussed in brief in this paper. This extracted information is then processed to analyze the traffic condition and send proper action commands to optimize the traffic making it faster, safer and efficient.
\end{abstract}

\section{Keywords}

IoT- internet of things, $5 \mathrm{G}-5^{\text {th }}$ generation, GSMA - global system of mobile communication, Ubidots platform.

\section{INTRODUCTION}

Internet of Things is changing people's lives. Each day more and more devices and objects are getting connected to some sort of network, whether it is a coffee maker, an umbrella, security camera, air quality sensors or automated cars etc. , each and every area of life will be implementing IoT in one way or the other.

Internet of things refers to devices connected to the internet which are able to transmit data to the cloud network from where the data is processed and returned. This also promotes and contributes to big data world.

This paper focuses on the unique features and benefits of $5 \mathrm{G}$ over other technologies in the world of IoT. It will also go on and discuss some IoT solutions which can be possible through existing cellular technologies like $3 \mathrm{G}$ and $4 \mathrm{G}$ networks.

According to a GSMA intelligence forecast report, 25.5 billion devices would be connected to IoT by 2025 , out of which 3.1 billion devices will Low powered wireless mobile IoT networks (like 6LoWPAN etc.) [1].

In todays IoT scenario a variety of wireless technologies are used which are short ranged as well as wide ranged. The short range including unlicensed spectrum like $\mathrm{WiFi}$, Bluetooth, ZigBee and wide area technologies include GSM, LTE and $5 \mathrm{G}$.

The LTE or 4G network will co-exist with its descendent (5G)

Offering its services until $5 \mathrm{G}$ expands globally in the upcoming years[2].
Though 4G runs the current world of IoT very well, 5G comes with a number of advantages like supporting a large number of static and mobile IoT devices, each having different speed and bandwidth requirements.

\section{5G SOLUTIONS FOR IOT}

As discussed in the earlier sections $5 \mathrm{G}$ provides better solutions for IoT as compared to presently used LTE network and previously used other networks.

The 1st generation (1G) introduced voice communication.

The 2 nd generation added texting then came $3 \mathrm{G}$ adding data to its predecessors then $4 \mathrm{G}(\mathrm{LTE})$ has everything $3 \mathrm{G}$ has but faster.

And now 5G is enters the picture and will be even faster.

To give you an idea of what kind of speed, talk about, downloading a full length HD movie in seconds.

But $5 \mathrm{G}$ is about lot more than just speed.

\subsection{Low Latency}

Latency is the time delay before the data starts being transferred following the instructions for its transfer.

The low latency that $5 \mathrm{G}$ promises is of key importance to many machines and devises which are to be connected through IoT .

Especially helpful in the case of machines which run by themselves i.e. autonomous machines and controlling industrial machines and devices will be optimized significantly.

This is because a low latency through $5 \mathrm{G}$ will allow the systems to process substantial amount of dynamic data in real time.

\section{IOT AIDED TRAFFIC MANAGEMENT SYSTEM}

With the smart city initiative was launched in India under the name "SMART CITIES MISSION" with the goal to establish viable cities that offer core infrastructure and a decent and clean environment to the residents.

One of the objective of the initiative is to ensure public safety on convenience. The IoT aided traffic management system's ensures a smoother, faster and safer traffic with increased traffic flow and decreased congestion.

With the advancement of technology, today devices such as cameras are very compact and high quality and available at 
cheap prices. Hard ware advances allows real time processing through multicore and parallel processing.

Detection of vehicles will be done through imaging technology [3]enabling on road vehicle detection. The multicore and parallel processing will allow real time vehicle detection.

The logic behind traffic detection is - the sensors (i.e. cameras) integrated with computers through cloud database and wireless network provides information, which is then processed through control algorithms to manage and predict traffic. The traffic light is mounted with a camera aided with image processing modules to obtain image sequences of the road and provide data for analysis and computation to calculate the and optimize the traffic[4].

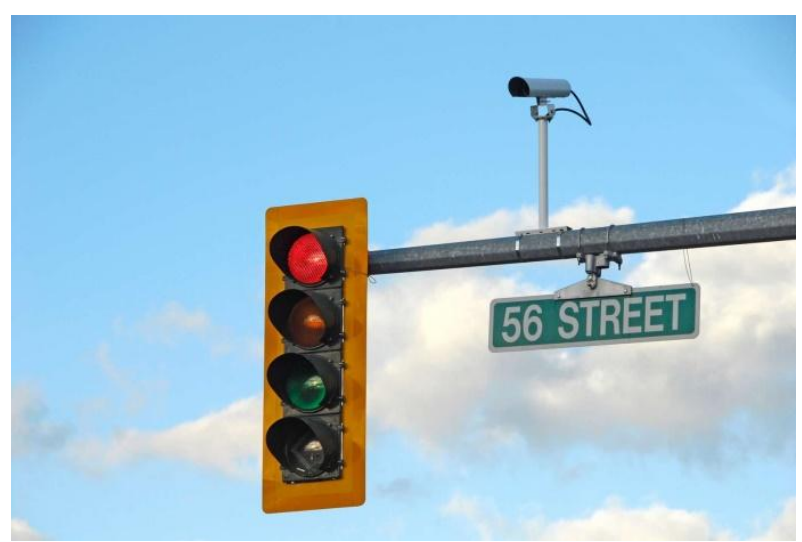

Fig. 1 - Traffic Light Mounted with a Camera

The Ubidots platform sends the images to the cloud server.

An adaptive neuro fuzzy controller sends a control signal which is used coordinate the traffic signal.

\section{HOW DETECTION OF VEHICLES WORK}

CCTV or closed circuit television cameras are used for surveillance. Detection of moving vehicle, classification of the detected vehicle and counting of the no. of vehicle is done using this system.

Detection can take place through several methods :

1. Background subtraction method.

2. Frame differencing and motion based methods.

3. Feature based method

\subsection{Background Subtraction Method}

In this method, the foreground objects (i.e. the vehicles) are extracted from the stored background frame from the surveillance video. This is one of the most widely used methods for vehicle detection.

A lot of techniques can be used for background subtraction method, many of which work on the Gaussian probability distribution model for each pixel in the frame. When the pixel values are updated by the Gaussian probability distribution model[6-10], each pixel (x,y) in the frame is categorized or identified either as a part of the foreground ( vehicles) or as a part of the background. A formula (1) is used for this identification

Let, $\mathrm{I}(\mathrm{x}, \mathrm{y})$ be the intensity of the pixel,
$\mathrm{C}$ be a constant,

$\operatorname{Mean}(\mathrm{x}, \mathrm{y})$ be the mean

Std $(\mathrm{x}, \mathrm{y})$ be the standard deviation

Then, the formula used is

$\mathrm{I}(\mathrm{x}, \mathrm{y})-\operatorname{Mean}(\mathrm{x}, \mathrm{y})<(\mathrm{C} * \operatorname{Std}(\mathrm{x}, \mathrm{y}))$------- (1) [11]

The background detection method does suffer a disadvantage in case of change in lighting and weather conditions[5].

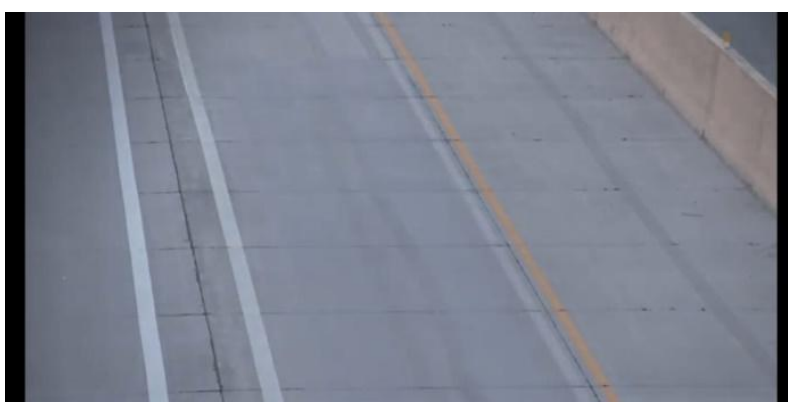

Fig.2- background image/frame[4]

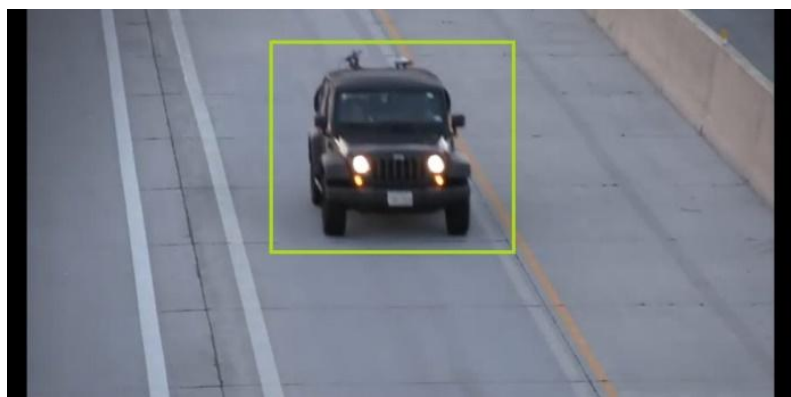

Fig. 3-vehicle detected through background subtraction[4]

\subsection{Frame Difference and Motion based Methods}

In this method, two subsequent frames in the image sequence are subtracted to differentiate the vehicles from the background image. The motion is detected and traced by isolating the foreground (moving) object referred as "blobs" through a sequence of assigned, aligned and analyzed pixels to different class of objects based on the speed and orientation of the movements of the objects with respect to the background image or frame $[5,12,13,14]$.

\subsection{Feature based Methods}

This method focuses on the movements of the various sub features of the foreground moving objects like the edges, the head lights, the tail light. These set of features are detected and further analyzed for subsequent frames hence analyzing and calculating the traffic speed and flow. This method also solves one of the problems posed by background subtraction method i.e. of vehicle overlapping[15].

\section{METHODOLOGY AND BLOCK DIAGRAM}

The steps involved in traffic optimization are[4]:

1. Development of an Adaptive Neuro Fuzzy traffic light controller which is hinge on the density of the traffic and the waiting time of the vehicles. This can be built using platforms like NI Multisim or MATLAB SIMULINK 
2. Transferring the image sequence captured by the surveillance camera to cloud using Ubidots platform and Arduino UNO.

3. Providing the calculated control action signal to the traffic lights

(This paper only discuss the detection through detection using background subtraction method.)

In the conventional traffic light controller, the lights change at a constant time cycle. This conventional system cannot provide a optimal solution for traffic management. This is where an adaptive traffic light control system comes in.

The camera sensor captures image sequence of a traffic lane, this data is then sent to the cloud where it is analyzed. The data is processed by image processing methods, in this case,

background subtraction method. The traffic congestion data is extracted from the image sequence.After that, the extracted information is fed to the MATLAB adaptive neuro fuzzy traffic controller.

The background subtraction method faces a setback due to change in lighting condition.

$\rightarrow$ This problem is solved by providing 2 or more different set of background model to the SIMULINK model, for different lighting conditions say day and night time. The SIMULINK model extracts the "blobs" or blocks of pixels and calculated the magnitude and the direction of the motion of each input unit.

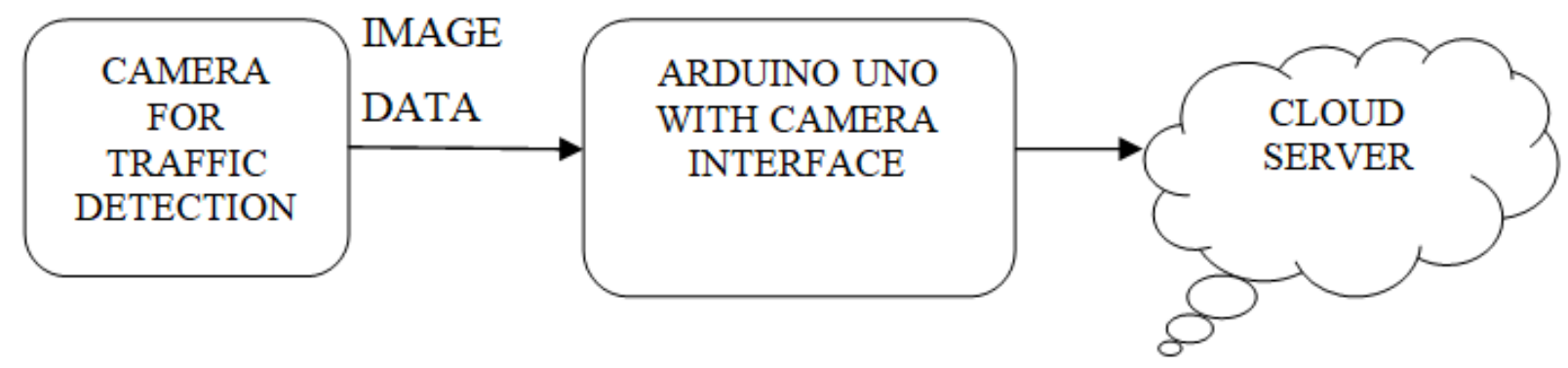

Fig. 4- Block Diagram of the system

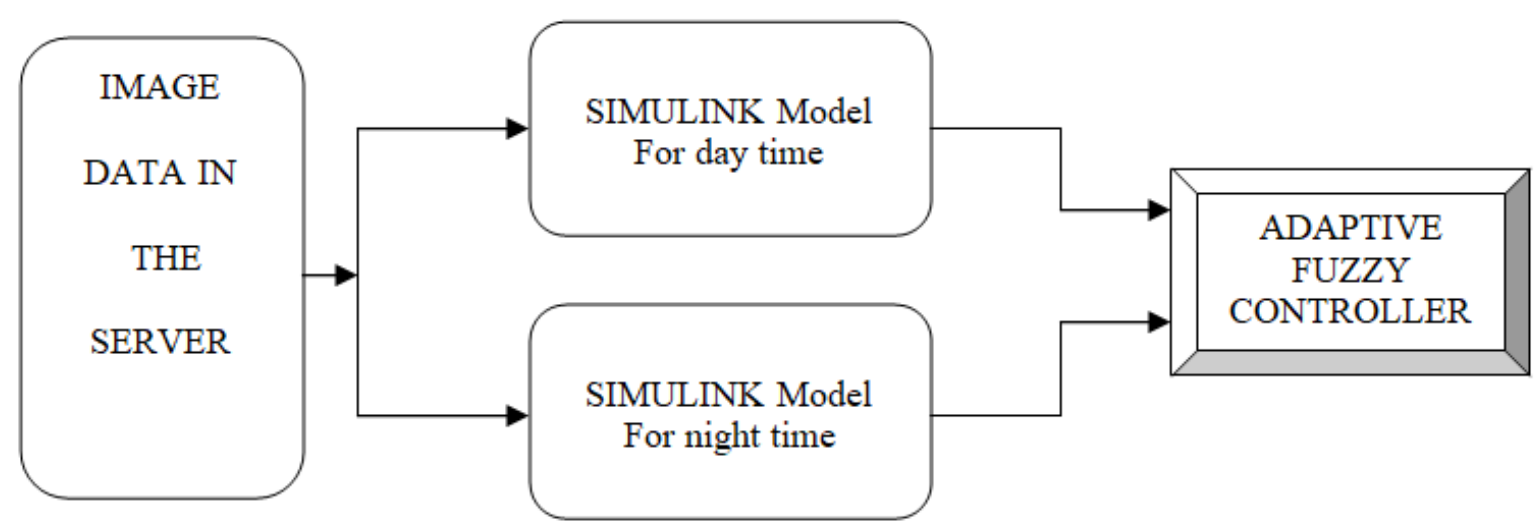

Fig. 5- Block Diagram of system in cloud

$\rightarrow \quad$ For calculating the speed of the vehicle, the centroid of the vehicle is detected, the distance traveled by the vehicle is the movement of the vehicle across the frame. The time for which the vehicle is in the frame is the time period. Now the distance and the time are known and hence the speed of the vehicle is estimated.

The traffic density is calculated based on the no. of blobs detected in the lane. This technique is used in detection of vehicles during the day time, but cannot be used at night since the light conditions are not very good.

At night another method known as Ostu's method is used. In this method, the variance between the foreground and background classes is maximized. For this, the vehicle headlights are obtained as blobs. The density of the traffic is calculated by dividing the blob count by 2.[16]

The adaptive Neuro fuzzy Inference system makes use of a hybrid training method. This training method helps the system to automatically generate fuzzy rules according to given sets of input-output. Through this the ANFIS forms a structure for traffic control and then tuning and training is done[17].

A most suitable time for green light is indicated by the output depending upon the waiting time (input 1) and queue length (input 2).

$\rightarrow \quad$ The Ubidots is an IoT data analytics and visualization platform. It works in real time. One needs to create an account and a channel to store the data sent from the ubidots platform

$\rightarrow \quad$ Arduino UNO is an open source microcontroller board based with an atmega 328 processor. The arduino uno connect the system to a cloud server to collect, monitor and manage traffic situations through the web.

\section{CONCLUSION}

IoT based traffic system can significantly improve traffic conditions giving the user more control over traffic 
congestion and remote access to traffic status thus allowing the user to save a lot of valuable time. This traffic control system will also allow the police officers to view the traffic in real time. This system will also help in reducing accidents, making the roads safer for people. Such traffic control systems can be very complicated to make a good, feasible and a fast network is very important for smooth and flawless functioning. 5G brings the perfect solution for this problem, proving large no. of connections at a speed which makes the real time data processing possible for upcoming IoT projects and innovations.

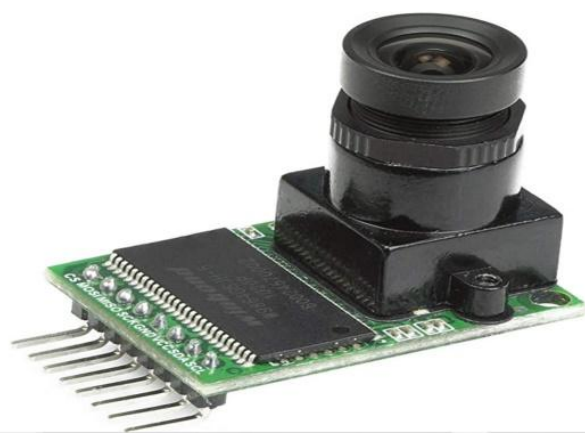

Fig. 5a. arduCAM

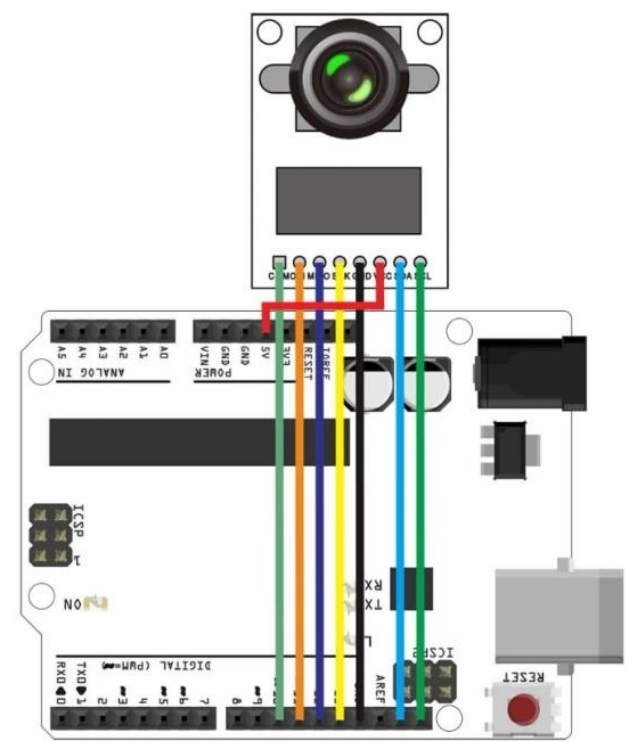

Fig. 5b. - camera connection for arduino UNO

The traffic control and detection system uses a camera sensor, an arduino board to send the collected data from the sensor to the cloud from where the data is processed in a adaptive neuro fuzzy system developed in MATLAB which computes and figures out the optimal and organized time frame for traffic lights.

\section{REFERENCES}

[1] https://www.gsma.com/iot/wpcontent/uploads/2018/08/GSMA-IoT-infographic_1819_2.png

[2] The Mobile Economy 2018, GSMA Intelligence

[3] Sudheer Kumar, Nithya Merin Jacob, Dr. Ciji Pearl Kurian, Dr. Kumara Shama, -Calibration curves for luminance data acquisition using Matlab\|, International Journal of Engineering Research and Technology, vol.3, no.3, ISSN: 2278-0181, 2014.
[4] Anna Merine George, V.I. George - IoT based smart traffic light control system, 2018 International Conference on Control, Power, Communication and Computing Technologies (ICCPCCT).

[5] L. Vasu, "An effective step to real-time implementation of accident detection system using image processing," Master of Science, Oklahoma State University, USA, 2010.

[6] C. R. Wren, et al., "Pfinder: real-time tracking of the human body," Pattern Analysis and Machine Intelligence, IEEE Transactions on, vol. 19, pp. 780-785, 1997.

[7] T. E. Boult, et al., "Frame-rate omnidirectional surveillance and tracking of camouflaged and occluded targets," in Visual Surveillance, 1999. Second IEEE Workshop on, (VS'99), 1999, pp. 48-55.

[8] G. Xiang, et al., "Error analysis of background adaption," in Computer Vision and Pattern Recognition, 2000. Proceedings. IEEE Conference on, 2000, pp. 503-510 vol.1

[9] I. Haritaoglu, et al., "W4: Real-Time Surveillance of People and Their Activities," Pattern Analysis and Machine Intelligence, IEEE Transactions on, vol. 22, pp. 809-830, 2000.

[10] K. Toyama, et al., "Wallflower: principles and practice of background maintenance," in Computer Vision, 1999. The Proceedings of the Seventh IEEE International Conference on, 1999, pp. 255-261 vol.1.

[11] Raad Ahmed Hadi, Ghazali Sulong and Loay Edwar George - VEHICLE DETECTION AND TRACKING TECHNIQUES: A CONCISE REVIEW- Signal \& Image Processing : An International Journal (SIPIJ) Vol.5, No.1, February 2014

[12] B. Han, et al., "Motion-segmentation-based change detection," SPIE Defence \& Security Symposium 2007, pp. 65680Q-65680Q, 2007.

[13] D. Koller, et al., "Towards robust automatic traffic scene analysis in real-time," in Decision and Control, 1994., Proceedings of the 33rd IEEE Conference on, 1994, pp. 3776-3781 vol.4

[14] W. Zhang, et al., "Moving vehicles detection based on adaptive motion histogram," Digit. Signal Process., vol. 20, pp. 793-805, 2010

[15] M. Xiaoxu and W. E. L. Grimson, "Edge-based rich representation for vehicle classification," in Computer Vision, 2005. ICCV 2005. Tenth IEEE International Conference on, 2005, pp. 1185-1192 Vol. 2.

[16] Anna Merine George, Palika Shetty S, -Fuzzy Controller for an Image based Traffic Systemll, International Journal of Management, IT and Engineering (IJMIE), vol.2, no.6, ISSN: 2249-0558, pp.291-305, 2012.

[17] Kingsley Monday Udofia, Joy Omoavowere Emagbetere, Frederick Obataimen Edeko, -Dynamic traffic signal phase sequencing for an isolated intersection using ANFIS\|, Automation, Control and Intelligent Systems, vol.2, no.2, pp.21-26, 2014. 